\title{
Photoemission and Optical Spectroscopy of i-ZnMgEr Quasicrystal
}

A. Suchodolskis ${ }^{a}$, W. Assmus ${ }^{b}$, G.-J. Babonas ${ }^{a}$,

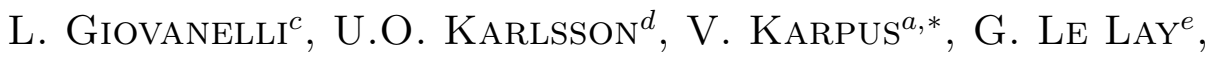
A. RËZA ${ }^{a}$ AND E. $\mathrm{UHRIG}^{b}$

${ }^{a}$ Semiconductor Physics Institute, A. Goštauto 11, 01108 Vilnius, Lithuania ${ }^{b}$ Physikalisches Institut, J.W. Goethe-Universität Robert-Mayer Str. 2-4, 60325 Frankfurt am Main, Germany ${ }^{c}$ TASC-INFM, S.S. $14 \mathrm{Km}$ 163.5, 34012 Basoviza, Trieste, Italy

${ }^{d}$ Department of Materials Physics, KTH P.O. Box Electrum 229, 16440 Kista, Sweden ${ }^{e}$ CRMC2-CNRS, Campus de Luminy Case 913, 13288 Marseille Cedex 9, France

The results of photoemission and optical studies of icosahedral single-grain ZnMgEr quasicrystals are presented. Synchrotron radiation photoemission measurements were performed on in situ cleaved samples at $10^{-10}$ mbar pressure and low, $140-150 \mathrm{~K}$ temperature. The valence band photoemission spectra measured reveal a simple metal-type valence band of icosahedral ZnMgEr with a distinct Fermi edge cut-off and with a sharp pseudogap feature. The photoemission spectra of shallow $\mathrm{Mg} 2 p$ and $\mathrm{Zn} 3 d$ core levels indicate a coordination shift of $\mathrm{Mg} 2 p$ spin-orbit doublet and an oscillating dependence of $\mathrm{Zn} 3 d$ photoemission line components on the photon energy within the $h \nu=20-160 \mathrm{eV}$ range investigated. The optical response of icosahedral $\mathrm{ZnMgEr}$, a dispersion of the dielectric function, was measured by the spectroscopic ellipsometry technique in the $0.5-5.0 \mathrm{eV}$ spectral range. The optical spectra of the quasicrystal indicate the Drude-type optical response superimposed with a peak of interband optical transitions. The interband optical transitions can be modelled with the pseudogap parameters which agree with those deduced in an analysis of the photoemission valence band spectra.

PACS numbers: 71.23.Ft

\section{Introduction}

Photoemission and optical spectroscopies of quasicrystals (QC's) are the main tools for determination of their electronic structure. The electronic structure

*corresponding author; e-mail: karpus@pfi.lt 
of QC's is an intriguing, unsolved problem of their physics and various theoretical models of electron quantum states in QC's are discussed in literature: from the Bloch-type extended states to the localized and the critical ones.

Here we present results of photoemission (PE) and optical studies of an icosahedral (i-) ZnMgEr quasicrystal, which show that the QC valence band is predominantly due to the $s$ (or $s p$ ) derived electron states and is similar to valence bands of simple metals. The experimental data confirm the "band structure hypothesis", within which the electronic subsystem of quasicrystals is treated as the nearly free electron (NFE) gas, affected by a weak quasicrystalline potential. An analysis of the experimental data shows that the i-ZnMgEr energy spectrum in the vicinity of the Fermi level is determined by the intersections of the Fermi surface with the 222100 and 311111 Bragg planes.

The suggested model of the i-ZnMgEr energy spectrum fits both the experimental PE valence band spectrum and the experimental optical spectrum of the dielectric function.

\section{Experimental}

The icosahedral $\mathrm{Zn}_{65} \mathrm{Mg}_{24} \mathrm{Er}_{11}$ single-grain quasicrystals were grown by the liquid-encapsulated top-seeded solution-growth method. The structural perfection of the QC's was confirmed by the sharp, resolution limited Bragg peaks of their diffraction patterns.

Photoemission measurements were performed with the angle-resolved scanning photoelectron microscope, at beamline BL31 of the Swedish synchrotron-radiation facility MAX-lab (Lund). The specimens for PE measurements were prepared in a form of small, $\approx 0.5 \mathrm{~cm}$ long rods with $\approx 1 \mathrm{~mm}^{2}$ cross-section. The samples were cleaved in situ at $10^{-10}$ mbar and low, 140-150 K temperature. The incident photon beam was focussed to the $1.5 \mu \mathrm{m}$ spot on the cleaved sample surface at the normal incidence and the PE spectra were recorded with the VG CLAM2 analyser mounted at $47.5^{\circ}$. The acceptance angle of the analyser is $\approx 6^{\circ}$ at the photon energy of $40 \mathrm{eV}$ and $\approx 10^{\circ}$ at $h \nu=100 \mathrm{eV}$. The cleanliness of the cleaved surfaces was routinely monitored following an evolution of photoemission from the O $2 p$ states [1].

Optical measurements of the dielectric function $\varepsilon(\omega)$ were carried out in ambient and at a room temperature by the spectroscopic ellipsometry technique. Prior to the optical measurements the i-ZnMgEr samples were mechanically polished to remove an oxide layer [2]. The $\varepsilon(\omega)$ function in the VI-UV range of $c a .0 .5-5 \mathrm{eV}$ was measured making use of the photometric ellipsometer with rotating analyser at the incidence angle of $80^{\circ}$.

\section{Valence band PE spectrum}

Figure 1 presents an experimental valence band (VB) photoemission spectra of $\mathrm{i}-\mathrm{ZnMgEr}$ recorded at the incident photon energies of $c a .45 \mathrm{eV}$ and the tem- 
perature of $150 \mathrm{~K}$. As seen, the valence band bottom is dominated by the $\mathrm{Zn} 3 d$ shallow core level doublet. The broad complex structure at binding energies below ca. $5 \mathrm{eV}$ is due to the Er $4 f_{7 / 2}$ states, as can be identified from a comparison with the PE spectra of pure erbium [3].

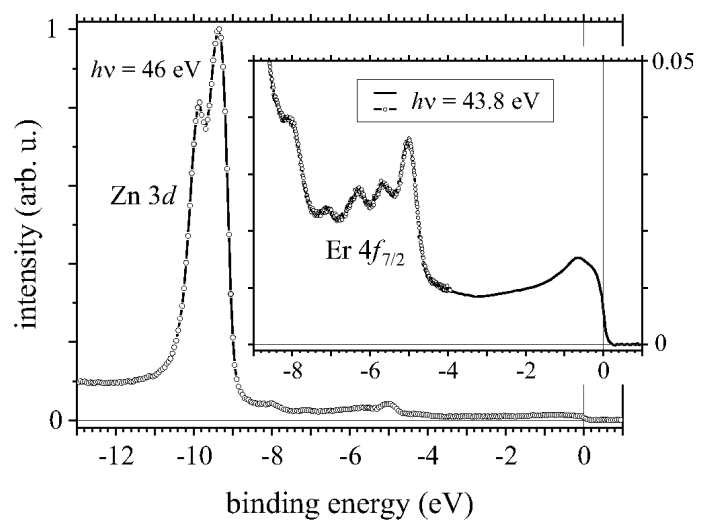

Fig. 1. Valence band PE spectra of $\mathrm{i}-\mathrm{ZnMgEr}(T=150 \mathrm{~K})$. Inset shows PE spectra from 0 to $-8 \mathrm{eV}$ binding energy measured at higher magnification.

As seen from Fig. 1, the valence band PE spectrum in the vicinity of the Fermi level shows a distinct pseudogap feature. The spectral intensity exhibits a maximum at $c a .0 .7 \mathrm{eV}$ below the Fermi level and a subsequent decrease as $\varepsilon_{\mathrm{F}}$ is approached. The VB spectra in the vicinity of the Fermi level and below it down to $\operatorname{Er} 4 f_{7 / 2}$ line have not changed in their spectral shape while recording the spectra at different photon energies in the interval of $20-60 \mathrm{eV}$. Additionally, the $\mathrm{i}-\mathrm{ZnMgEr} \mathrm{VB}$ spectrum in the interval of binding energies down to $\mathrm{ca} .4 \mathrm{eV}$ is rather close to $\mathrm{VB}$ spectra of $\mathrm{i}-\mathrm{ZnMgY}$ and $\mathrm{i}-\mathrm{ZnMgHo}$ quasicrystals [4]. This allows us to conclude that the $\mathrm{i}-\mathrm{ZnMgEr} \mathrm{VB}$ at the Fermi level and below it down to $c a .4 \mathrm{eV}$ is determined primarily by the $s$ (or $s p$ ) derived electron states.

An analysis of the near Fermi-edge PE spectrum, carried out following the scheme of our previous analysis [4] of the $\mathrm{i}-\mathrm{ZnMg}(\mathrm{Y}, \mathrm{Ho}) \mathrm{PE}$ spectra, shows [5] that the $\mathrm{i}-\mathrm{ZnMgEr}$ energy spectrum in the vicinity of the Fermi level is determined by intersections of the Fermi surface with the (222100) and (311111) Bragg planes, the corresponding reciprocal lattice half-vectors of which $\frac{1}{2} g_{222100}=1.53 \times 10^{8} \mathrm{~cm}^{-1}$ and $\frac{1}{2} g_{311111}=1.59 \times 10^{8} \mathrm{~cm}^{-1}$ (determined from i-ZnMgEr diffraction patterns) lie in a close proximity to the Fermi wave vector $k_{\mathrm{F}}=1.49 \times 10^{8} \mathrm{~cm}^{-1}$. The intersections open the $\Delta_{222100}=0.64 \mathrm{eV}$ and $\Delta_{311111}=1.25 \mathrm{eV}$ pseudogaps in the energy spectrum and lead to the van Hove singularities in the density of states at the pseudogap edges, which are smoothed in the experimental PE spectrum by the instrumental energy resolution $0.2 \mathrm{eV}$ of the experimental PE setup used and by the intrinsic broadening of electron states $\Gamma=0.24 \mathrm{eV}$. The Fermi level $\varepsilon_{\mathrm{F}}=9.32 \mathrm{eV}$, as determined from PE spectra analysis, is located above the $\Delta_{222100}$ 
pseudogap and lies within the $\Delta_{311111}$ pseudogap. The feature of the experimental $\mathrm{PE}$ spectra at $-0.7 \mathrm{eV}$ is due first of all to the $\Delta_{222100}$ pseudogap and is affected by the lower edge of the $\Delta_{311111}$ pseudogap.

\section{Shallow core levels}

The photoemission spectra of the shallow $\mathrm{Mg} 2 p$ core level in i-ZnMgEr recorded at several different photon energies are presented in Fig. 2a. The underlying structure of the $\mathrm{Mg} 2 p \mathrm{PE}$ line is the spin-orbit doublet, as is evident from a comparison of the $\mathrm{Mg} 2 p$ spectrum in $\mathrm{i}-\mathrm{ZnMgEr} \mathrm{QC}$ with that in $\mathrm{Zn}_{2} \mathrm{Mg}$ single crystal (full-dotted curve in Fig. 2a). The magnesium dizinc is the usual crystalline hexagonal compound and was studied as a reference system, because it is close to $\mathrm{i}-\mathrm{ZnMgRE}(\mathrm{RE}=$ rare earth) quasicrystals in its atomic composition and in some respects in the local atomic surrounding (see, e. g., [6]). As seen from Fig. 2a, the $\mathrm{Mg} 2 p$ line in i-ZnMgEr QC is much broader than that in $\mathrm{Zn}_{2} \mathrm{Mg}$. The
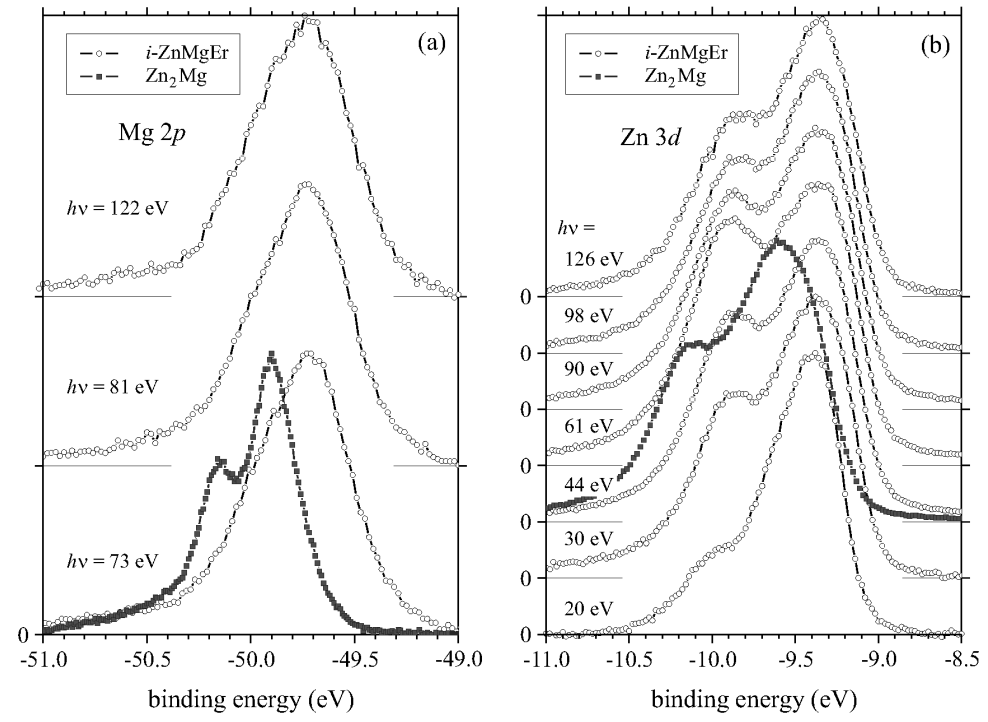

Fig. 2. PE spectra of (a) Mg $2 p$ and (b) Zn $3 d$ shallow core level in i-ZnMgEr quasicrystal (open dots, $T=140-150 \mathrm{~K}$ ) and in crystalline $\mathrm{Zn}_{2} \mathrm{Mg}$ (full dots, $T \approx 100 \mathrm{~K}$ ).

Mg $2 p$ PE spectra of i-ZnMgEr can be well approximated by two binding-energy shifted spin-orbit doublets with the constant intensity ratio for all photon energies investigated. This suggests that the broad $\mathrm{Mg} 2 p$ line in i-ZnMgEr is due to different inequivalent magnesium sites in quasicrystalline lattice. The coordination shift of the core level deduced is $c a .0 .1-0.2 \mathrm{eV}$.

The i-ZnMgEr PE spectra of the shallow Zn $3 d$ core level recorded at different photon energies are presented in Fig. $2 \mathrm{~b}$ and show a distinct doublet structure of the $\mathrm{Zn} 3 d \mathrm{PE}$ line with the binding energy displacement of $\approx 0.55 \mathrm{eV}$. Let us note 
that contrary to the $\mathrm{Mg} 2 p \mathrm{PE}$ line the $\mathrm{Zn} 3 d$ one in $\mathrm{i}-\mathrm{ZnMgEr}$ is not broadened as compared to the $\mathrm{Zn} 3 d \mathrm{PE}$ line in magnesium dizinc (full-dotted curve in Fig. 2b). This can be due to a formation of the finite-width $\mathrm{Zn} 3 d$ energy bands. Since the average distance between $\mathrm{Zn}$ atoms in the $\mathrm{QC}$ is larger than that in $\mathrm{Zn}_{2} \mathrm{Mg}$, the eventual Zn $3 d$ energy bands in $\mathrm{i}-\mathrm{ZnMgEr}$ should be narrower, as expected from the tight-binding band structure model.

Though, as can be traced from $44 \mathrm{eV}$ and $61 \mathrm{eV}$ PE spectra in Fig. 2b, the $\mathrm{Zn} 3 d \mathrm{PE}$ line of i-ZnMgEr has a more complex structure than a simple doublet, the main feature observed is an obvious variation of the intensity ratio of the primary components of the doublet. To reveal the effect we modelled the $\mathrm{Zn} 3 d \mathrm{PE}$ spectra by two simple Doniach-Šunjić lines with the same line-width and asymmetry parameters and the constant binding energy displacement. The deduced dependence of the intensity ratio $\beta$ of the components on the photon energy is presented in Fig. 3. A variation of the ratio of $\mathrm{Zn} 3 d \mathrm{PE}$ doublet components

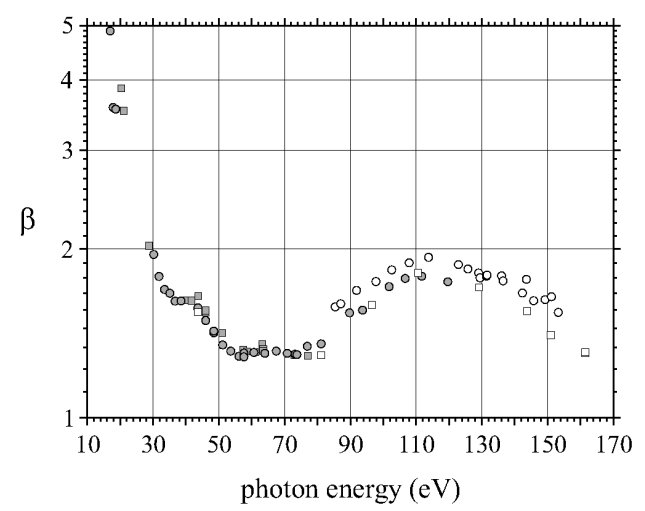

Fig. 3. Dependence of the ratio of the $\mathrm{Zn} 3 d$ shallow core level PE doublet components in $\mathrm{i}-\mathrm{ZnMgEr}$ on the photon energy. Different dots correspond to $\mathrm{Zn} 3 d \mathrm{PE}$ spectra recorded from various i-ZnMgEr specimens.

within the $h \nu$ interval of $c a .20-70 \mathrm{eV}$ has been previously observed by Himpsel et al. [7] for the pure crystalline $\mathrm{Zn} \mathrm{(0001)} \mathrm{and} \mathrm{has} \mathrm{been} \mathrm{interpreted} \mathrm{as} \mathrm{due} \mathrm{to} \mathrm{the}$ dispersion of $\mathrm{Zn} 3 d$ energy bands. However, the oscillating character of the $\beta(h \nu)$ dependence observed at higher photon energies for i-ZnMgEr (Fig. 3) can be also attributed to the interference effects of the final electron states, i.e. to the inner diffraction of photoelectrons on the quasicrystalline potential field.

\section{Optical response}

The dielectric function $\varepsilon(\omega)$ of i-ZnMgEr, measured by spectroscopic ellipsometry technique, is presented in Fig. 4. The $\varepsilon(\omega)$ dispersion is close to that measured previously for $\mathrm{i}-\mathrm{ZnMgY}$ quasicrystal [8] and shows a superposition of the Drude-type optical response and a contribution of the interband transitions. 


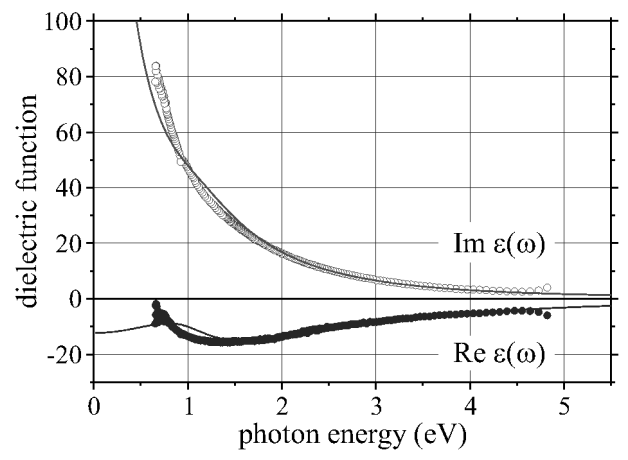

Fig. 4. Dispersion of the dielectric function of i-ZnMgEr quasicrystal.

The latter obviously manifests itself in the real part of $\varepsilon(\omega)$, which has a spectral feature at $c a .1 \mathrm{eV}$.

To model the experimental $\varepsilon(\omega)$ dispersion the dielectric function was presented in the form

$$
\varepsilon(\omega)=1+\mathrm{i} \frac{4 \pi}{\omega}\left[\sigma_{\text {Drude }}(\omega)+\sigma_{\mathrm{ib}}(\omega)\right],
$$

where $\sigma_{\text {Drude }}(\omega)$ is the Drude optical conductivity,

$$
\varepsilon_{\text {Drude }}(\omega)=\frac{\sigma_{\mathrm{dc}}}{1-\mathrm{i} \omega \tau}
$$

$\sigma_{\mathrm{dc}}$ is the static electric conductivity and $\sigma_{\mathrm{ib}}(\omega)$ is the optical conductivity due to the interband transitions. The optical conductivity due to the interband transitions across a pseudogap at the intersection of an isoenergetic surface with the Bragg planes can be calculated analytically following Ashcroft and Sturm algorithm [9] at given values of the $g$ vector of the Bragg planes, their multiplicity $N$, the pseudogap $\Delta$, the Fermi energy $\varepsilon_{\mathrm{F}}$, and the broadening parameter $\Gamma_{\mathrm{opt}}$.

Assuming that the optical interband transitions within the photon range investigated are due first of all to $\Delta_{311111}$ pseudogap, we simulated the experimental dielectric function at the following set of parameters: $\sigma_{\mathrm{dc}}=6270 \Omega^{-1} \mathrm{~cm}^{-1}$, $\hbar / \tau=1.9 \mathrm{eV}, \Delta_{311111}=1 \mathrm{eV}, \varepsilon_{\mathrm{F}}=9.35 \mathrm{eV}$, and $\Gamma_{\mathrm{opt}}=0.46 \mathrm{eV}$. The results of the calculations are presented by curves in Fig. 4 and, as seen, approximate the experimental $\varepsilon(\omega)$ dispersion. The estimated value of the static conductivity $6270 \Omega^{-1} \mathrm{~cm}^{-1}$ is close to that measured by Fisher et al. [10], $\approx 5900 \Omega^{-1} \mathrm{~cm}^{-1}$, while the value of the plasma frequency $\omega_{\mathrm{p}} \approx 11 \mathrm{eV}$, determined by applying the oscillator sum rule $\int_{0}^{\infty} \mathrm{d} \omega \operatorname{Re} \sigma(\omega)=\omega_{\mathrm{p}}^{2} / 8$, is comparable with the $\omega_{\mathrm{p}} \approx 8.3 \mathrm{eV}$ value, determined by Chernikov et al. [11] for the i-ZnMgY and i-ZnMgHo quasicrystals from an analysis of their reflectivity spectra.

The partial contributions of the Drude and the interband transitions are shown in Fig. 5, which presents the real part of the optical conductivity $\sigma(\omega)=$ $\sigma_{\text {Drude }}(\omega)+\sigma_{\mathrm{ib}}(\omega)$, and in the figure inset, where the real and imaginary parts 


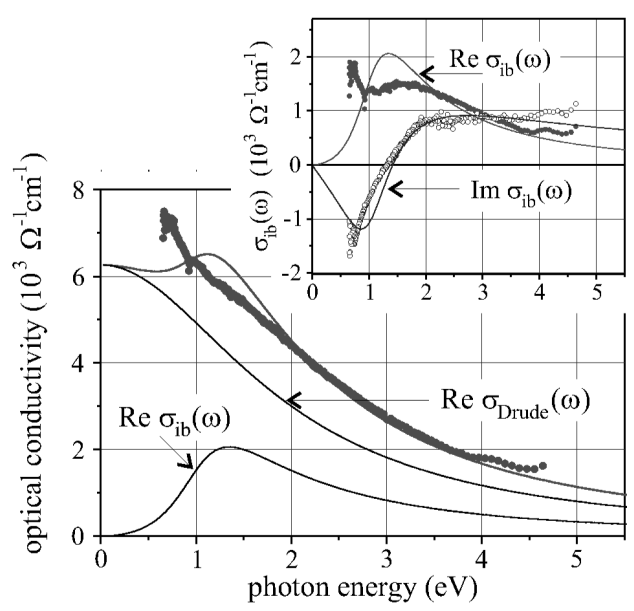

Fig. 5. Optical conductivity of i-ZnMgEr quasicrystal.

of $\sigma_{\mathrm{ib}}(\omega)$ are presented. As seen, both the real and imaginary parts of interband optical conductivity are approximated in general trends by the NFE model used, and, thus, the optical data support the assumption of the $s p$ origin of i- $\mathrm{ZnMgEr}$ valence band. It should be noted that the $\Delta_{311111}=1 \mathrm{eV}$ and $\Gamma_{\text {opt }}=0.46 \mathrm{eV}$ values of the pseudogap and its broadening, which were used in simulation of the optical data, are close to those, $\Delta_{311111}=1.25 \mathrm{eV}$ and $\Gamma=0.24 \mathrm{eV}$, deduced from the PE spectra analysis. This confirms the logical consistency of the energy spectrum model employed.

\section{Conclusions}

Summarizing we conclude that the photoemission and the optical response of icosahedral ZnMgEr quasicrystal show the i-ZnMgEr valence band in the vicinity of the Fermi level and below it down to $c a .4 \mathrm{eV}$ to be of the simple-metal type and to originate predominantly from the $s p$-electron states. The density of states nearby the Fermi level is determined by intersections of the Fermi surface with the 222100 and 311111 Bragg planes.

The experimental valence band PE spectra and a dispersion of the dielectric function can be approximated within NFE model. The approximation reproduces both the PE data in the vicinity of the Fermi level and the main experimental optical $\varepsilon(\omega)$ features. The pseudogap parameters evaluated from the PE spectra and from the optical data are close to each other.

The photoemission spectra of shallow $\mathrm{Mg} 2 p$ and $\mathrm{Zn} 3 d$ core levels indicate a coordination shift of $\mathrm{Mg} 2 p$ spin-orbit doublet and an oscillating dependence of Zn $3 d \mathrm{PE}$ line components on the photon energy. 


\section{Acknowledgments}

Support by the EC Access to Research Infrastructure programme and the Swedish Institute is gratefully acknowledged.

\section{References}

[1] A. Suchodolskis, W. Assmus, B. Čechavičius, J. Dalmas, L. Giovanelli, M. Göthelid, U.O. Karlsson, V. Karpus, G. Le Lay, R. Sterzel, E. Uhrig, Appl. Surf. Sci. 212-213, 485 (2003).

[2] V. Karpus, A. Rėza, A. Suchodolskis, G.J. Babonas, W. Assmus, R. Sterzel, V. Kazlauskienè, J. Miškinis, A. Miniotas, Mater. Sci. (Medžiagotyra) 6, 148 (2000).

[3] F. Gerken, Ph.D. thesis, Universität Hamburg, 1982, p. 90.

[4] A. Suchodolskis, W. Assmus, L. Giovanelli, U.O. Karlsson, V. Karpus, G. Le Lay, R. Sterzel, E. Uhrig, Phys. Rev. B 68, 054207 (2003).

[5] A. Suchodolskis, W. Assmus, L. Giovanelli, U.O. Karlsson, V. Karpus, G. Le Lay, E. Uhrig, to be published.

[6] N. Tamura, M. Beyss, K. Urban, Philos. Mag. Lett. 74, 89 (1996).

[7] F.J. Himpsel, D.E. Eastman, E.E. Koch, A.R. Williams, Phys. Rev. B 22, 4604 (1980).

[8] V. Karpus, G.-J. Babonas, A. Rèza, W. Assmus, R. Sterzel, Lithuanian J. Phys. 40, 118 (2000).

[9] N.W. Ashcroft, K. Sturm, Phys. Rev. B 3, 1898 (1971).

[10] I.R. Fisher, Z. Islam, A.F. Panchula, K.O. Cheon, M.J. Kramer, P.C. Canfield, A.I. Goldman, Philos. Mag. B 77, 1601 (1998).

[11] M.A. Chernikov, S. Paschen, E. Felder, P. Vorburger, B. Ruzicka, L. Degiorgi, H.R. Ott, I.R. Fisher, P.C. Canfield, Phys. Rev. B 62, 262 (2000). 